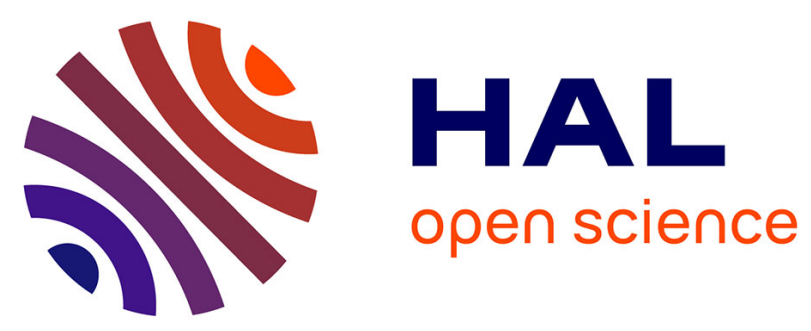

\title{
Electronic Structure and Bistable Conformational Study of the Tetraphenylporphyrin Erbium(III) Acetylacetonate Complex on the CaF2/Si(100) Surface at Low Temperatures
}

Yitao Wang, Anne-Gaëlle Boyer, Hélène Sauriat-Dorizon, Eric Duverger, Damien Riedel

\section{To cite this version:}

Yitao Wang, Anne-Gaëlle Boyer, Hélène Sauriat-Dorizon, Eric Duverger, Damien Riedel. Electronic Structure and Bistable Conformational Study of the Tetraphenylporphyrin Erbium(III) Acetylacetonate Complex on the CaF2/Si(100) Surface at Low Temperatures. Journal of Physical Chemistry C, 2021, 125 (26), pp.14453 - 14460. 10.1021/acs.jpcc.1c02943 . hal-03306247

\section{HAL Id: hal-03306247 \\ https://hal.science/hal-03306247}

Submitted on 29 Jul 2021

HAL is a multi-disciplinary open access archive for the deposit and dissemination of scientific research documents, whether they are published or not. The documents may come from teaching and research institutions in France or abroad, or from public or private research centers.
L'archive ouverte pluridisciplinaire HAL, est destinée au dépôt et à la diffusion de documents scientifiques de niveau recherche, publiés ou non, émanant des établissements d'enseignement et de recherche français ou étrangers, des laboratoires publics ou privés. 
This document is confidential and is proprietary to the American Chemical Society and its authors. Do not copy or disclose without written permission. If you have received this item in error, notify the sender and delete all copies.

\section{Electronic structure and bistable conformational study of tetraphenylporphyrin Erbium(III) acetylacetonate complex on the CaF2/Si(100) surface at low temperature}

\begin{tabular}{|c|c|}
\hline Journal: & The Journal of Physical Chemistry \\
\hline Manuscript ID & jp-2021-02943y.R2 \\
\hline Manuscript Type: & Article \\
\hline $\begin{array}{r}\text { Date Submitted by the } \\
\text { Author: }\end{array}$ & 04-Jun-2021 \\
\hline Complete List of Authors: & $\begin{array}{l}\text { Wang, Yitao; CNRS, Institut des Sciences Moléculaire d'Orsay } \\
\text { Boyer, Anne; Université Paris-Saclay, Institut de Chimie Moléculaire et } \\
\text { des Matériaux d'Orsay (ICMMO), CNRS, Univ. Paris Sud, Université Paris- } \\
\text { Saclay, F-91405 Orsay, France. } \\
\text { Sauriat-Dorizon, Hélène; ICMMO } \\
\text { Duverger, Eric; FEMTO-ST, Université de Franche Comté } \\
\text { Riedel, Damien; CNRS, Institut des Sciences Moléculaire d'Orsay }\end{array}$ \\
\hline
\end{tabular}

\section{SCHOLARONE" Manuscripts}




\title{
Electronic Structure and Bistable Conformational Study of Tetraphenylporphyrin-Erbium(III) Acetylacetonate Complex on the $\mathrm{CaF}_{2} / \mathrm{Si}(100)$ Surface at Low Temperature
}

\author{
Yitao Wang ${ }^{1}$, Anne-Gaëlle Boyer ${ }^{2}$, Hélène Sauriat-Dorizon ${ }^{2}$, Eric Duverger ${ }^{3}$, Damien \\ Riedel $^{1 *}$ \\ ${ }^{1}$ Institut des Sciences Moléculaires d'Orsay (ISMO), CNRS, Univ. Paris Sud, Université Paris-Saclay, \\ F-91405 Orsay, France. \\ ${ }^{2}$ Institut de Chimie Moléculaire et des Matériaux d'Orsay (ICMMO), CNRS, Univ. Paris Sud, Université \\ Paris-Saclay, F-91405 Orsay, France. \\ ${ }^{3}$ Institut FEMTO-ST, Univ. Bourgogne Franche-Comté, CNRS, 15B avenue des Montboucons, F-25030 \\ Besançon, France.
}

\begin{abstract}
:
The synthesis of tetraphenylporphyrinate Erbium(III) acethylacetonate (acac) complexes is realized and their properties studied at the nanoscale when adsorbed on a semi-insulating $\mathrm{CaF}_{2} / \mathrm{Si}(100)$ surface. Our findings reveal that the ErTPP-(acac) molecules can adsorb in two main on-site conformations. Following precisely located $\mathrm{dI} / \mathrm{dV}$ measurements at various specific positions (phenyls, pyrroles, Er-(acac)), the relative locations of the Er cation and the apical ligand (acac) can be deciphered for each observed conformation. Hence, one of the adsorbate conformations presents the acac ligand parallel to the porphyrin plane with the Er atom outside the macrocycle plane. The second conformation is related to what is known in the gas phase where the acac ligand is oriented vertically on top of the $\mathrm{Er}$ atom. This work is combined with a theoretical investigation that uses density functional theory methods to bring into light details of the two observed conformations. Additional proofs of our discoveries are related to the vibrational excitation of the ErTPP-(acac). A comparison with a theoretical estimation of the vibrational modes reveals how the electronic resonance near the valance band edge of the insulting layer is suitable to distinguish between the two adsorbed conformations.
\end{abstract}

Keywords: Lanthanide porphyrin molecules, silicon; molecular manipulation, scanning tunneling microscopy, molecular electronics.

*damien.riedel@universite-paris-saclay.fr 


\section{INTRODUCTION}

Molecular species containing lanthanide (Ln) atoms have attracted specific attention for decades. These last years, in particular, have emerged strong motivations to study four-fold symmetry molecules having rare earth atom center including Terbium, Dysprosium or Ytterbium to target the study of new types of magnetic molecules ${ }^{1}$ or special light emitters. ${ }^{2}$ In this framework, several studies have been focusing on an efficient caging of the electronic structure of the Ln atom via the formation of double-decker structures., ${ }^{3,4}$ Other magnetic properties dealing with the Kondo effect are also investigated via the formation of controlled $2 \mathrm{D}$ molecular lattices. ${ }^{6}$ In addition to their magnetic properties, Ln atoms allow specific electronic or optical excitation, thanks to the longer lifetime of the Ln $4 \mathrm{f}$ orbitals compared to $3 \mathrm{~d}$ and $5 \mathrm{~d}$ orbitals of transition metals. As a counterpart, one of the major drawbacks of such magnetic molecular structures deals with the compromise between accessing $4 \mathrm{f}$ orbitals of the central atoms and the ability to preserve its electronic structure from its surroundings. ${ }^{7,8}$ The investigation of single metallated phthalocyanines and porphyrins molecules has revealed the possibility to partly tune their electronic and optical properties by changing the central metal atom..$^{9,10,11,12,13}$ So far, while self-assembled Ln-Pc or Ln-TPP molecules have been investigated in the gas phase ${ }^{14}$, single molecule are rarely studied on surfaces at the atomic scale. ${ }^{15}$

In this work, we investigate the electronic structure of single tetraphenylporphyrinate Erbium (III) acethylacetonate complex, i.e. ErTPP-(acac) molecules on the $\mathrm{CaF}_{2} / \mathrm{Si}(100)$ surface at $9 \mathrm{~K}$ via scanning probe techniques. $\mathrm{dI} / \mathrm{dV}$ spectroscopy performed at different locations on the molecule allows describing the electronic structure of the two observed conformations. These results are compared with calculations that use the density functional theory to reproduce the STM images and the spectroscopic curves. Our findings reveal that the two observed conformations could be switched between each other via the position change of the central Er atom and the apical ligand.

\section{METHODS}

\section{Chemical methods}


The metalation reaction of porphyrin free base with Erbium(III) lanthanide cation is performed by treating tetraphenylporphyrin $\left(\mathrm{H}_{2} \mathrm{TPP}\right)$ with an excess of $\operatorname{Er}(\mathrm{acac})_{3} \cdot 3 \mathrm{H}_{2} \mathrm{O}$ in melted imidazole under argon. Progress of the reaction is monitored by following the appearance of the Soret band of the metalloporphyrin at $424 \mathrm{~nm}$ and by the increase of the Q-band absorbance at $553 \mathrm{~nm}$, corresponding to $\pi-\pi^{*}$ electronic transitions. The resulting crude product is purified on aluminum oxide column in methanol and precipitated in a mixture of acetone/water to afford in a moderate yield the neutral $\operatorname{Er}(\mathrm{III})(\mathrm{TPP})(\mathrm{L})(\mathrm{S}) \mathrm{x}$. The electrospray ionization mass spectrum of the complex shows the corresponding mass peak of $\operatorname{Er}(\mathrm{III})(\mathrm{TPP})(\mathrm{acac})$ as well as the presence of other mass peaks. Their analysis highlights the presence of different Erbium(III)TPP complexes in which the nature of the apical ligand (L) varies. Indeed, at high temperature, the acetylacetonate (acac) ligand pyrolyzes in a major pyrolytic product, the acetate $(\mathrm{OAc}=\mathrm{OCOCH} 3)$, that can coordinate to the lanthanide ion and form the acetate-coordinated porphyrin, $\operatorname{Er}(\mathrm{III})(\mathrm{TPP})(\mathrm{OAc}) .{ }^{16,17}$ Besides, the Erbium porphyrin complex with free acetylacetonate is also observed in the mass spectrum, in which lanthanide are coordinated by many molecules of solvent (e.g. water, methanol) that stabilize the metallic cation.

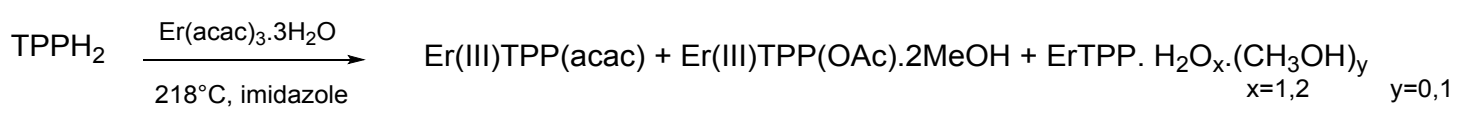

A quantity of $0.1 \mathrm{~g}$ of porphyrin $(0,163 \mathrm{mmol}, 1 \mathrm{eq})$ was heated at $218^{\circ} \mathrm{C}$ with $0.378 \mathrm{~g}$ of $\mathrm{Er}(\mathrm{acac}) 3.3 \mathrm{H}_{2} \mathrm{O}(0.814 \mathrm{mmol}, 5 \mathrm{eq})$ and $5 \mathrm{~g}$ of imidazole for two days, under inert atmosphere. The reaction is monitored by UV-visible spectroscopy and stopped after the shift of the Soret band at 424 $\mathrm{cm}^{-1}$ as well as the disappearance of the $\mathrm{Q}$ bands at 518 and $648 \mathrm{~nm}$. The mixture is then dissolved with ethanol and water at room temperature, extracted three times with dichloromethane and organic phases were concentrated. The crude is purified on a neutral aluminum oxide column in a mixture of dichloromethane and methanol $(100 / 0$ to $95 / 5 ; \mathrm{v} / \mathrm{v})$. The product is then precipitated in acetone and 
water to afford $32 \mathrm{mg}$ of ErTPP-(acac). The positive electrospray (ESI) mass spectrum of $\mathrm{C}_{49} \mathrm{H}_{36} \mathrm{ErN}_{4} \mathrm{O}_{2}$ is given in the ESI document in table S1.

\section{STM methods}

The STM experiments are performed with ultra-high vacuum (UHV) scanning tunneling microscopy (CREATEC) working at low temperature $(9 \mathrm{~K})$. The silicon samples used in our experiments (from ITME) are n-type doped (As, $\rho=5 \mathrm{~m} \Omega . \mathrm{cm}$ ) and cut along the (100) plane. The bare $\mathrm{Si}(100)$ surface is firstly prepared via two main steps in UHV environment. The silicon surface is firstly cleaned by resistive heating at $1220 \mathrm{~K}$ to remove the oxide layer. Then, the silicon surface is reconstructed by several cycles of flash ( $\sim 5 \mathrm{~s})$ heating followed by an annealing period of 2 to $3 \mathrm{~min}$. The cleaned silicon sample is then kept at $\sim 1000 \mathrm{~K}$ and placed in front of an effusion cell in which pure $\mathrm{CaF}_{2}$ crystals are heated in an $\mathrm{HOPG}$ crucible at $1240 \mathrm{~K}$ for about 2 to 4 minutes. During the epitaxy of the $\operatorname{Si}(100)$ surface, the base pressure in the UHV chamber is kept under $9 \times 10^{-11}$ Torr. A detailed description of the obtained semi-insulating layer is provided in previous works. ${ }^{18,19}$ Following the $\mathrm{Si}(100)$ surface epitaxy with $\mathrm{CaF}_{2}$, the sample is cooled down to $\sim 12 \mathrm{~K}$ prior to the adsorption of the ErTPP-(acac) molecule. Subsequently, the ErTPP-(acac) molecule are sublimated at a temperature of $496 \mathrm{~K}$ on the obtained surface during a very short time (very few seconds). The sample is then inserted at $12 \mathrm{~K}$ in the STM chamber for analysis.

The used STM tips are made from a tungsten wire electrochemically etched in a $\mathrm{NaOH}$ solution and then cleaned via electron bombardment in the UHV preparation chamber. The scanning tunneling spectroscopy (STS) curves obtained in our experiments arise from two lock-in amplifiers. Their results are compared to avoid spurious $\mathrm{dI} / \mathrm{dV}$ peaks and to reduce the background noise. During the STS measurements, an amplitude modulation of $5-10 \mathrm{mV}$ at a frequency of $833 \mathrm{~Hz}$ is applied on the sample. The tunnel current is converted into a voltage signal outside the STM chamber with a noise level below $7 \mathrm{mV}$ giving a robust average signal-to-noise ratio on the measured $(\mathrm{dI} / \mathrm{dV}) /(\mathrm{I} / \mathrm{V})$ signals. During the measurement, all the $(\mathrm{dI} / \mathrm{dV}) /(\mathrm{I} / \mathrm{V})$ curves are acquired at various STM tip heights for estimating the 
possible energy shifts of the DOS peaks due to band-bending effects induced by the STM tips or the voltage drop arising from the double barrier junction..$^{20}$

\section{Theoretical methods}

The ErTPP-(acac) molecule has been studied by the DFT method using the Siesta computer code. $^{21,22,23,24}$ Two molecular structures are considered. The two molecules are the ErTPP-(acac) (i.e. 5,10,15,20-tetraphenylporphyrin (TPP) with an acetylacetonate apical ligand with two different positions of their apical ligand. ${ }^{25,26}$ The calculations are carried out using a polarized double $\zeta$ basis set (DZP) and a nonlocal norm conserving pseudopotentials with relativistic methods that are necessary to reveal the properties of such heavy element. For the exchange correlation functional, we used the generalized gradient approximation (GGA) including the van der Waals interaction..$^{27,28,29}$ The mesh cutoff of 100 Ry with a single k-point at the center $\Gamma$ for the Brillouin zone integration was considered to calculate the total energies within a numerical precision of $1 \mathrm{meV}$. Geometry relaxation was performed by the conjugate-gradient method with the force convergence criterion of $0.02 \mathrm{eV} / \AA$ in the same slab volume (i.e., $4.64 \times 3.10 \times 4.44 \mathrm{~nm}^{3}$ ) corresponding at the $\mathrm{CaF}_{2} / \mathrm{Si}(100)$ slab. In a second step, to determine the best conformational/adsorption energy, SIESTA (Spanish Initiative for Electronic Simulations with Thousands of Atoms) DFT molecular dynamic simulation was performed. The initial configurations were calculated by random placement of the molecule on $\mathrm{CaF}_{2} / \mathrm{Si}(100)$ slab. Then, energy minimizations are performed on the system via a position relaxation calculation to obtain an optimized configuration of the molecule and the slab. The charge distributions between molecules and the slab surface are analyzed using the Bader approach. ${ }^{30,31}$ The vibrational spectroscopy of the ErTPP-(acac) is calculated by using the Gaussian 09 package with the hybrid B2LYP functional. The 6-311G* and the ANO-RO relativistic basis sets are used to relax the molecule and compute the IR spectrum.

\section{RESULTS AND DISCUSSION}


Considering the molecular synthesis methods used to obtain the desired ErTPP molecule, several adsorption sites are observed on the STM topographies. We have thus concentrated our study on the most often observed ErTPP-(acac) conformations, i.e. on the molecules showing STM topographies with fourfold symmetry and having a central area featuring the presence of an apical ligand. As recall in Figures. $1 \mathrm{a}$ and $1 \mathrm{~b}$, the lateral size of the Er(III)TPP-(acac) molecule in the gas phase is $13 \AA$ while its height, compared to the macrocycle, reaches $\sim 5 \AA$ due to the presence of the acetylacetonate-apical fragment. As shown in Figures. 1c and 1d, the size of the two observed molecular conformations A and $\mathrm{B}$ are in good agreement with the calculated ones.

As a consequence of the presence of an apical ligand, the occupied states STM topographies ($2.5 \mathrm{~V})$ acquired on top of the $\operatorname{Er}(\mathrm{III}) \mathrm{TPP}-(\mathrm{acac})$ exhibit clear instabilities in the STM topographies, especially, when the STM tip is scanning over the central part of the molecule (Figure. 1c). As well, a second conformation B can be observed on STM topography in Figure. ld where no more scanning instabilities are observed. From the STM topographies in Figures. 1c and 1d, one can check that the lateral sizes of the adsorbed conformations are in coherence with the ones of the ErTPP-(acac) as in gas phase (Figures. 1a).
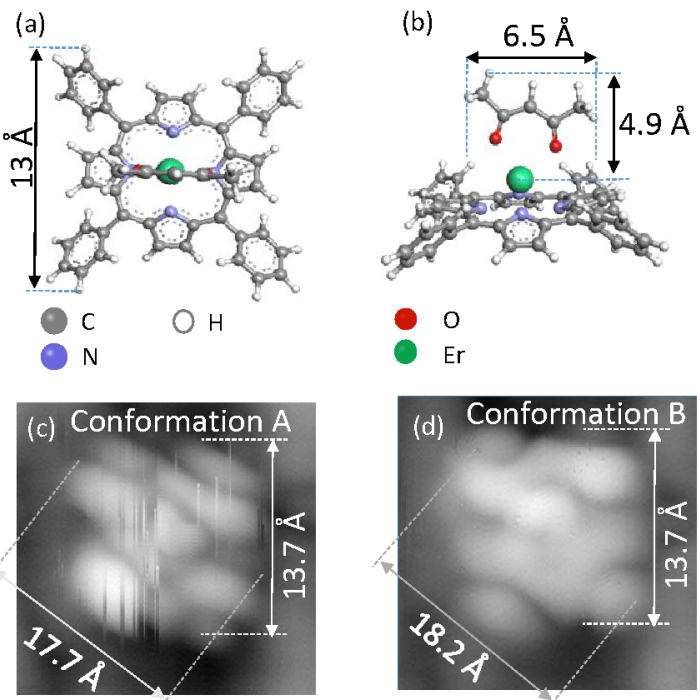

\footnotetext{
Figure 1: Top (a) and (b) side balls-and-stick representations of the ErTPP-(acac) molecule with one apical acetylacetone ligand. The grey, red, white, green and blue balls represent the carbon, oxygen, hydrogen, erbium and nitrogen atoms, respectively. (c) and (d) $(22 \times 22$ $\AA^{2}$ ) STM topography $\left(V_{s}=-2.5 \mathrm{~V}, \mathrm{I}=18 \mathrm{pA}\right)$ of two ErTPP-(acac) conformations $A$ and $\mathrm{B}$, respectively, observed after adsorption on the $\mathrm{CaF}_{2} / \mathrm{Si}(100)$ surface stripes.
} 
To investigate the properties of the two observed molecular conformations, we have performed various differential inelastic electron tunnel current spectroscopy (IETS) measurements at several positions over the molecule. The selected IETS positions over the first conformation A (Figure. 1c) are described in Figure. 2a while probing the four phenyls ( $\mathrm{Ph} 1$ to $\mathrm{Ph} 4)$ and four pyrroles (Py1 to Py4) rings as well as the center (Ce) of the molecule. The $\mathrm{dI} / \mathrm{dV}$ curves in Figures $2 \mathrm{~b}$ and $2 \mathrm{c}$ exhibit one main peak of density of state (DOS) centered at $\sim-1.9 \mathrm{~V}$ in the occupied states as well as another peak of DOS, of lower intensity, centered at $\sim 1.9 \mathrm{~V}$ in the unoccupied state. In the central part of the molecule, the $\mathrm{dI} / \mathrm{dV}$ spectrum (Figure. 2d) shows one intense peak of DOS, likewise centered at $\sim-2.0 \mathrm{~V}$, with a second superimposed $\mathrm{d} / / \mathrm{dV}$ curve that suddenly stops at $-1.7 \mathrm{~V}$, signature of the observed instability at the center of the molecule in Figure. 2a.

(a)

Conformation A
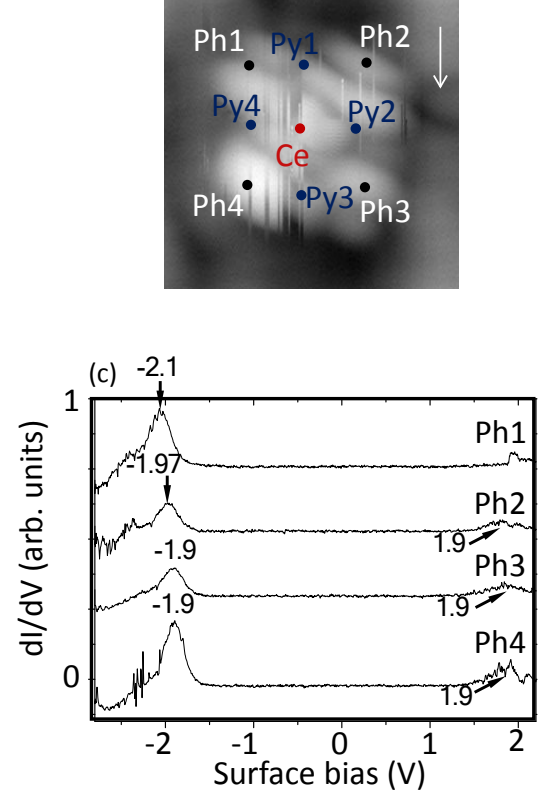

(b)

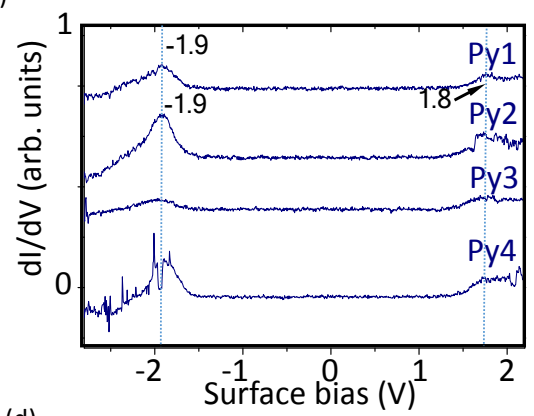

(d)

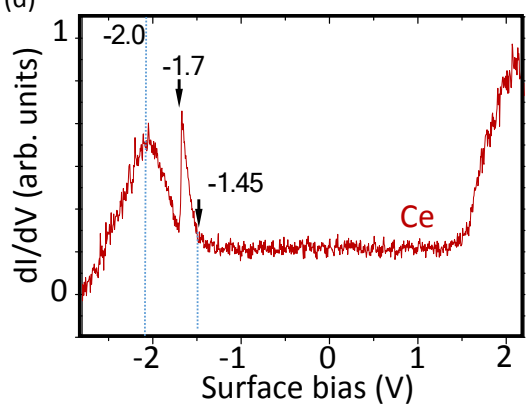

Figure 2: (a) $22 \times 22 \mathrm{~A}^{2}$ STM topography of the ErTPP molecule in the conformation A. The dots indicate where the dl/dV spectroscopy curves have been acquired. (b) to (d) dl/dV curves acquired on the four phenyls (b), the four pyrroles (c) and the central (d) positions on the ErTPP molecule as detailed in (a).

When the electronic structure of the ErTPP-(acac) molecule is probed at various specific molecular positions, the ensemble of the $\mathrm{dI} / \mathrm{dV}$ curves acquired on the conformation A (Figure. 2a) is very similar. This electronic structure is significantly different from what can be measured when $\mathrm{dI} / \mathrm{dV}$ 
is performed on a metallated tetraphenyl porphyrin molecules having no apical ligand ([M]TPP with M $=$ transition metal), on either a clean silicon surface ${ }^{32}$ or semi-insulating layer. ${ }^{33}$ In the gas phase, the energy of the frontiers orbitals HOMO and LUMO of neutral [M]TPP molecules are known to be usually located at $\sim 1.6 \mathrm{eV}$ on both sides of their electrochemical potential energy, while the phenyl groups exhibit occupied orbitals at higher energies (i.e. -2.5 to $-2.7 \mathrm{eV}$ ) with usually, stronger DOS intensities. The spatial DOS distribution of the frontiers orbitals is expected to be located along the porphyrin macrocycle, namely within the pyrrole groups of the molecules. Depending on the dihedral angles between the phenyl lateral ligands and the porphyrin plane, electronic coupling between the phenyl rings and the porphyrin macrocycle can be tuned leading to a spatial delocalization of their respective $\mathrm{DOS}^{33}$. In the present case, on a semi-insulating surface, the observed $\mathrm{dI} / \mathrm{dV}$ acquired on ErTPP-(acac) are expected to show $\mathrm{d} / \mathrm{dV}$ spectrum similar to the one in the gas phase with transition metals. However, one can also expect that the presence of the apical ligand and the lanthanide atom may play an important role in the known electronic structures.

To explain the dI/dV measurements observed in Figure. 2, we have acquired several STM images in constant height $(\mathrm{CH})$ mode, at the resonance energy of the observed $\mathrm{dI} / \mathrm{dV}$ peak maximum ($2.0 \mathrm{~V}$ ) when scanning over the same molecular conformation A observed in Figure. 2a. The $\mathrm{CH}$ mode allows, in our particular conditions in which the apical ligand is supposed to be located at a relatively high position between the porphyrin macrocycle and the STM tip, to reduce the interaction with the ligand and hence observe its instabilities at a lower frequency. The sequence of STM images shown in Figure. 3 has been obtained by scanning several times over the same molecule (Figure. 2a) with a height variation of - $0.9 \AA$ compared to the initial STM tip surface distance fixed by the used settings (i.e. -2.5 $\mathrm{V}, 14 \mathrm{pA}$ ). For the sake of clarity, we have added four dotted lines forming a quadrilateral joining each of the phenyl position on each STM image. Two additional blue median lines cross the geometrical central part form, dividing the molecule into four triangular zones. From a direct observation of these STM images, one can clearly observe a variation of brightness at the central area of the molecule. More precisely, while the brightness of the phenyls groups does not vary (except on the left part of the molecule), the bright feature at the middle of the molecule can move to four distinct positions. A careful 
look at the precise positions of the central bright feature indicates that it is located either on the right or left side of the lower triangle (Figure. $3 \mathrm{a}$ and $3 \mathrm{~b}$ ) or at the left and right side of the upper triangle (Figure. $3 \mathrm{c}$ and $3 \mathrm{~d})$. Therefore, the moving central feature of the molecule is not observed in the left or right triangle of the quadrilateral. As we have noticed above, the inner molecular movements perturb mainly one phenyl ring during the $\mathrm{CH}$ mode STM images sequence (i.e. the one located at the lower left corner of the molecule).
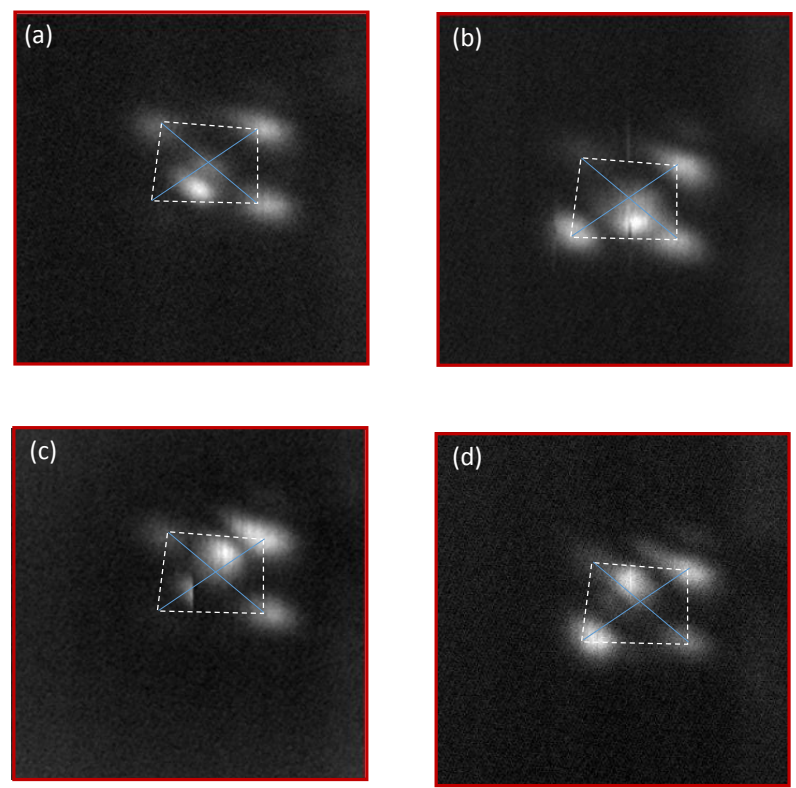

Figure 3: (a) to (d) $63 \times 63 \mathrm{~A}^{2}$ constant height mode STM images acquired on top of a ErTPP molecule in the conformation A at a constant bias of -2 Volts. The height of the STM tip apex is $0.5 \AA$ higher than the one fixed by the setting parameters $(\mathrm{Vs}=-2.5 \mathrm{~V}, \mathrm{I}=18 \mathrm{pA})$. The dotted lines are placed to help positioning the phenyls rings as well as the center of the molecule.

The intensity of the moving phenyl ring is significantly reduced (Figures. 3a and 3c) indicating that the ensuing phenyl group is probably having a slight rotational movement to compensate the steric hindrance of the inner apical ligand. This effect indicate that the acac is located, in the conformation A, at a rather short distance of the phenyl groups, i.e., lying down on the porphyrin. Note that from these observations, the apical ligand's motion of the ErTPP-(acac) can stabilize into four different positions in the conformation A. One of these sites is reproduced in the ESI document Figure. S1.

Following the observations of the ErTPP-(acac) in the conformation A (Figure. 2a), we have noticed that the excitation of this molecular conformation during the STM tip scanning at lower energies 
(ie $<-2.8 \mathrm{~V}$ ) allows to change and stabilize the appearance of the inner area of the molecule (Figure. 1d). The small surface defect observed on both molecular conformations (see the white arrows in Figures. $2 \mathrm{a}$ and $4 \mathrm{a}$ ) indicates that the molecule has not moved across the surface or along the stripes between the two observed conformational changes.

(a)

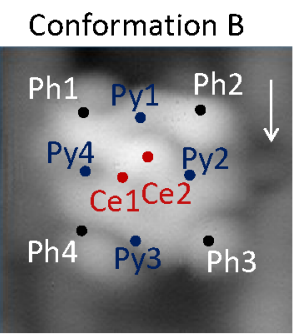

(c) -2.7

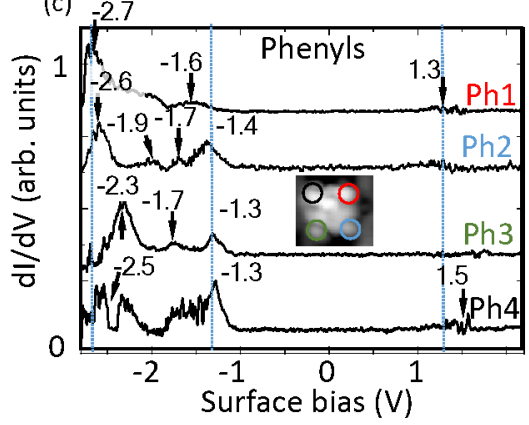

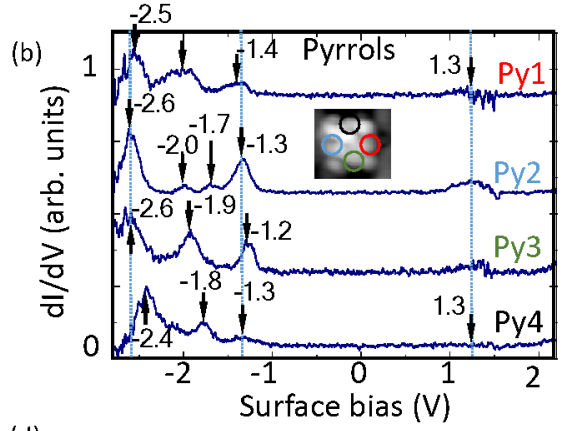

(d)

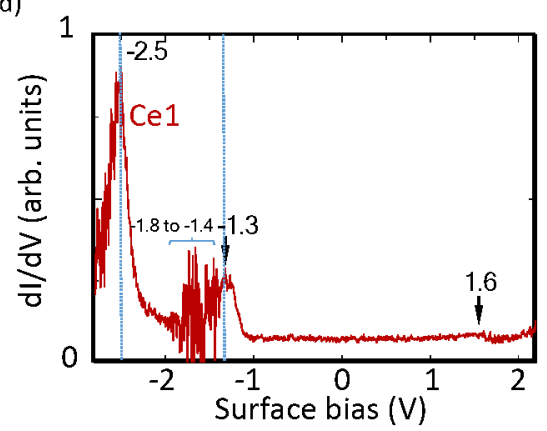

Figure 4: (a) $22 \times 22 \mathrm{~A}^{2}$ STM topography of the ErTPP molecule in the conformation $B$. The dots indicate where the dl/dV spectroscopy curves have been acquired. (b) to (d) $d / / d V$ curves acquired on the four phenyls (b), the four pyrroles (c) and the central (d) positions on the ErTPP molecule as detailed in (a). Typical maximums of the $\mathrm{dl} / \mathrm{dV}$ peaks are indicated with black arrows.

The molecular conformation B shown in Figure. $4 \mathrm{a}$ is probed at 9 different positions and the resulting $\mathrm{dI} / \mathrm{dV}$ normalized curves are presented in the panels $4 \mathrm{~b}$ to $4 \mathrm{~d}$. Each of the $\mathrm{dI} / \mathrm{dV}$ spectrum acquired on the phenyls and pyrroles groups are clearly different to the previous conformation. Indeed, one can observe several peaks of DOS centered at $-2.6 \mathrm{~V},-2.0 \mathrm{~V},-1.6 \mathrm{~V}$ and $-1.3 \mathrm{~V}$ for the occupied states and a DOS band spreading from 1.3 to $1.5 \mathrm{~V}$ for the unoccupied states. One should notice that this richness of peaks has not been observed yet in the measured $\mathrm{dI} / \mathrm{dV}$ curves acquired on other metallated tetraphenyl porphyrins. ${ }^{18,19,33}$ and seems to be specific to the ErTPP-(acac). Here, the fact that the DOS peaks are observed at similar energies over the phenyl and pyrrole groups indicates that a relatively strong delocalization of the DOS between the phenyls and the porphyrin macrocycle occurs. 
As mentioned above, this effect can be due to a larger dihedral angle between the phenyl rings and the plane of the porphyrin macrocycle compared to the one in the gas phase. This seems to apply for both conformations shown in Figure. 1c and 1d. Differently, the dI/dV spectra acquired at the central part of the molecule unveil only two types of DOS peaks. The ones having maximum energies observed at -2.5 $\mathrm{V}$ and $-1.3 \mathrm{~V}$, for the occupied states, and a single weak band of empty DOS state at $\sim 1.6 \mathrm{~V}$. One can also notice the noisy signal observed in the $\mathrm{dI} / \mathrm{dV}$ curve at the left shoulder of the DOS peak centered at $-1.3 \mathrm{~V}$ in Figure. $4 d$.

Our experimental observation and measurements of the ErTPP-(acac) on the semi-insulating $\mathrm{CaF}_{2} / \mathrm{Si}(100)$ surface could lead us to assume that the conformation B shown in Figure. 1d is the ErTPP molecule having lost the acetylacetonate apical ligand since the inner instabilities have disappeared under STM scanning. However, this process is unlikely since the energy required to induce this desorption can be rather high especially when considering the possible transition energy barrier to be overcomed (i.e. the binding energy of the $\mathrm{Er}-\mathrm{O}$ bond is $\sim 1.9 \mathrm{eV}$ ). In order to investigate the observed change between the conformations $\mathrm{A}$ and $\mathrm{B}$, we have performed numerical simulations based on the density functional method (DFT) of the ErTPP-(acac) molecular conformations when adsorbed on the semi-insulating ribbon of stripes (see the methods section 2.3 for more details). ${ }^{18}$ After the relaxation of the ErTPP-(acac) molecule, our DFT simulations indicate that two main conformations can be stabilized at the same adsorption site on the surface. Both of them are shown in the insets of Figures. $5 \mathrm{a}$ and $5 \mathrm{~b}$ where the ensuing projected density of state (PDOS) are reported for the pyrrole, phenyl and the central group of the molecule.

The calculated PDOS curves shown in Figure. 5a are the ones for the ErTPP-(acac) molecule in the conformation B, i.e., the same as the ones shown in Figures. 1a and $1 \mathrm{~b}$ where the acetylacetonate ligand stands up on top of the Er atom, the two oxygen atoms pointing towards the Er atom. In these conditions, the Er atom is located slightly higher than the porphyrin macrocycle. The corresponding PDOS curves in Figure. 5a bespeak that the phenyl groups have major peaks of PDOS for energies at $2.6 \mathrm{eV}$ and $2.5 \mathrm{eV}$ while the maximum energy of the frontiers orbitals are observed at $-1.3 \mathrm{eV}$ for the HOMO and $0.5 \mathrm{eV}$ for the LUMO. 


\begin{abstract}
Figure 5: (a) and (b) projected density of states (PDOS) curves calculated for the two conformations B and A respectively. The corresponding conformations are recalled in the insert of each set of curves. In (a), the $\mathrm{dl} / \mathrm{dV}$ curves are integrated over the sum of the four phenyls, four pyrroles, the Er atom and the acac fragment. In (b), the dl/dV curves are integrated over the four phenyls, four pyrroles and the Er atom.
\end{abstract}

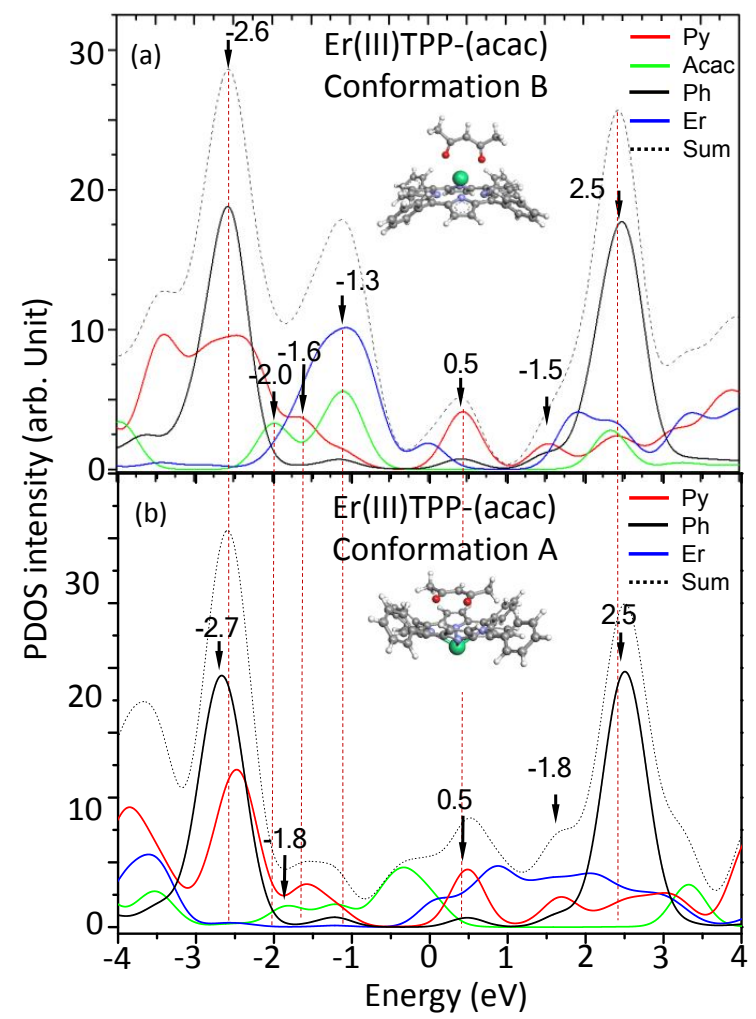

As explained previously for metalloporphyrin molecules with transition metals, the DOS of the frontiers orbitals are mainly located at the pyrrole groups (red curves at $0.5 \mathrm{eV}$ ) while the PDOS at the acac ligand combined to the one on the Er atom seems to play a central role in the PDOS peak at -1.3 $\mathrm{eV}$ (green and blue curves in Figure. 5a). We can also note the signature of the electronic coupling between the phenyl and pyrrole groups as the red curves (pyrroles) spreads out towards the PDOS peak of the phenyl groups.

The PDOS curves of the second relaxed conformation A of the ErTPP-(acac) molecule (Figure. 5b) are significantly different from the previous one due to two main aspects. Firstly, the position of the Er atom is moved down below the porphyrin macrocycle plane. Secondly, the acac ligand is now lying parallel to the porphyrin plane. A detailed description of both conformations is provided in Figure. S2 of the ESI document. From the PDOS curves in Figure. 5b, one can see that the new position of the Er atom below the porphyrin macrocycle has a strong impact on the calculated PDOS, especially in the occupied states, in the energy range varying from 0 to $-2 \mathrm{eV}$. Indeed, at this energy, most of the PDOS 
at the Er atom are clearly shifted to lower energies (blue curve peak at $-3.5 \mathrm{eV}$ ). Whereas, the PDOS curve of the acac (green curve) move to higher energies, i.e., in the energy band gap of the substrate, both forming a band of PDOS that spreads from $0.5 \mathrm{eV}$ to $-2.5 \mathrm{eV}$ that can interact with the PDOS of the pyrrole groups. Each curves in Figures. 5a and 5b exhibits sharp peaks of PDOS for the phenyl rings at similar energies for the two considered conformations A and B.

One can now compare the PDOS curves in Figure. 5 with the dI/dV curves described in Figures. 2 and 4. The PDOS peaks observed at $-1.3 \mathrm{eV},-1.6 \mathrm{eV}$ and $-2.0 \mathrm{eV}$ in Figure. $5 \mathrm{a}$ are very similar to the $\mathrm{dI} / \mathrm{dV}$ peaks observed in the occupied states at $-1.3 \mathrm{~V},-1.7 \mathrm{~V}$ or $-1.9 \mathrm{~V}$ in Figure. 4 . As well, the PDOS peak of the phenyl groups calculated at $-2.6 \mathrm{eV}$ is similar to the $\mathrm{dI} / \mathrm{dV}$ peak measured in the range -2.7 $\mathrm{V}--2.5 \mathrm{~V}$ (Figures. 4 and 5a). For the unoccupied state, the comparison is straightforward since only one band is observed in the range $1.3 \mathrm{~V}-1.5 \mathrm{~V}$ in the $\mathrm{dI} / \mathrm{dV}$ curves in Figure. 4. It is thus related to the PDOS peak at $2.5 \mathrm{eV}$ and its shoulder at $1.5 \mathrm{eV}$ in Figure. 5a. Similarly, the unoccupied dI/dV band observed in the curves in Figure. 2 at $1.8 \mathrm{~V}-1.9 \mathrm{~V}$ is associated with the PDOS shouldering calculated at $1.8 \mathrm{eV}$ in Figure. 5b. Note that the PDOS peak at $0.5 \mathrm{eV}$ can hardly be measured in the $\mathrm{dI} / \mathrm{dV}$ curves in Figure. 4 since the band gap energy of the $\mathrm{CaF}_{2}$ semi-insulating layer lies from $-1.5 \mathrm{~V}$ to $2 \mathrm{~V} .{ }^{18}$

Therefore, this comparison indicates that the conformation B observed on the STM topography in Figure. 1d can be attributed to the ErTPP-(acac) molecule having the apical ligand standing up on the Er atom (see inset in Figure. 5a). This is clarified by the relative strong electronic coupling between the acac ligand and the Er atom, especially around $1.3 \mathrm{eV}$ below the Fermi level energy $\left(\mathrm{E}_{\mathrm{f}}\right)$.

In the case of the STM topography observed in Figure. 1c showing an unstable central area, the ensuing ErTPP-(acac) conformation A can be attributed to the one shown in the inset in Figure. 5b. As mentioned previously, this conformation has a decreased electronic coupling between the Er atom and the acac ligand due to the motion of the Er atom below the porphyrin macrocycle plan. Therefore, when the STM tip scans over the molecule in this conformation, the contribution of the density of state of the acac ligand is preeminent and thus enhance the $\mathrm{dI} / \mathrm{dV}$ peak at an energy $\sim 2 \mathrm{eV}$ below the Fermi energy. Furthermore, because the acac fragment is less bonded to the Er atom in the conformation A, the ligand 
is freer to move on top of the molecule, which explains the series of constant height mode image shown in Figure. 3.
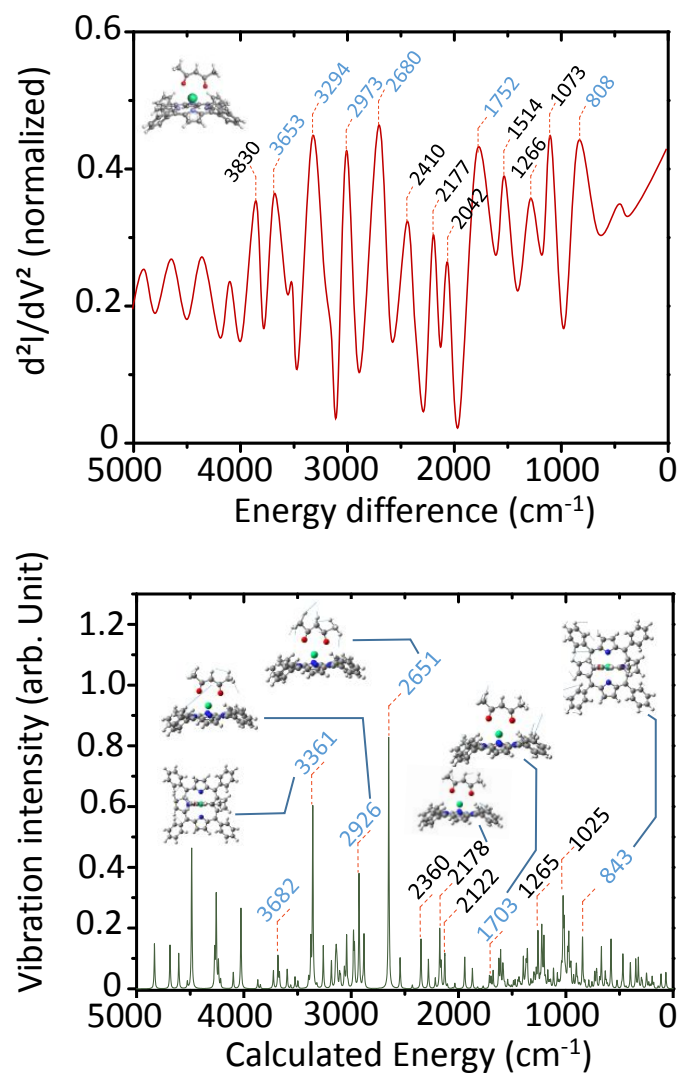

Figure 6: (a) $d^{2} I / d V^{2}$ curve calculated from a section of the acquired dl/dV spectrum at the Ce position on the conformation B of the ErTPP-(acac) molecule from Fig. $4 \mathrm{~d}$. The origin of the curve $(0 \mathrm{~cm}-1)$ is set at the resonance energy maximum of the DOS peak observed at $-1.3 \mathrm{eV}$. (b) Calculated vibrational spectra of the ErTPP-(acac) as in the conformation B in the gas phase (see method). The calculated energy values printed in blue are the most comparable to the ones measured in (a).

Since the geometrical and electronic aspects of the two observed ErTPP-(acac) conformations have been identified, we can now focus on the intriguing noisy signal that appears at the edge of the resonant peak at $-1.3 \mathrm{~V}$ in the $\mathrm{dI} / \mathrm{dV}$ curve in Figure. $4 \mathrm{~d}$. A detailed aspect of this signal is presented in Figure. $6 \mathrm{a}$, in which the energy difference is now considered from the maximum energy of the $\mathrm{dI} / \mathrm{dV}$ peak (ie $-1.3 \mathrm{eV}$ ) and where the calculated second derivative $\mathrm{d}^{2} \mathrm{I} / \mathrm{dV}^{2}$ is converted into $\mathrm{cm}^{-1}$. Similar effects have been already studied with various molecules on others insulating layers such as naphthalocyanine adsorbed on $\mathrm{Al}_{2} \mathrm{O}_{3} / \mathrm{NiAl}(110)^{34}$ or 2,5-bis(3-dodecylthiophen-2-yl)thieno[3,2-b]thiophene (BTTT) molecule adsorbed on $\mathrm{MoS}_{2} / \mathrm{Au}(111) \cdot{ }^{35}$ In both cases, the observed dI/dV oscillations 
at the edge of a DOS resonance peak arise from various types of vibrational excitations of the electronically excited molecule via the formation of a cation or anion. The relative high life time of the ionic species is supposed to be long enough to allow these observations. In the present case, the excited resonance of the ErTPP-(acac) is observed at $1.3 \mathrm{eV}$ below the Fermi level energy of the substrate. This energy is above the threshold valence band edge energy ( $1.5 \mathrm{eV}$ below $\left.\mathrm{E}_{\mathrm{f}}\right)$ of the $\mathrm{CaF}_{2} / \mathrm{Si}(100)$ measured previously. ${ }^{18}$ The averaged measured linewidth (FWHM) of the ionic resonance peak at $-1.3 \mathrm{~V}$ (not shown in Figure. 4d) is $\sim 110 \mathrm{meV}$. This gives an estimated lifetime of the ionic molecule of a few picoseconds. To compare our experimental data in Figure. 6a with theoretical estimations of the infrared excitation spectrum of the ErTPP-(acac), we have performed the frequency calculation of the vibrational spectra of this molecule in the gas phase using the Gaussian calculation package (see the method section 2.3 ). The obtained calculated spectrum represented in Figure. $6 \mathrm{~b}$ exhibit several vibrational peaks of highest intensity. If one compares the energy of the calculated peaks with the experimental data in Figure. $6 a$, six $d^{2} I / d V^{2}$ peaks can be attributed to the calculated ones with an average energy difference as small as $49 \mathrm{meV}$. Among these vibrational peaks, the ones in the range $800 \mathrm{~cm}^{-1}-2300 \mathrm{~cm}^{-1}$ are fairly related to the stretching mode of the pyrrole groups while those lying in the range $2500 \mathrm{~cm}^{-1}-$ $4000 \mathrm{~cm}^{-1}$ involve the apical ligand of the ErTPP-(acac). A detailed view of the stretching directions is provided in Figure. S3 of the ESI document. Different spectra have been calculated previously for other metalated porphyrins such as FeTPP. ${ }^{36}$ Their comparison with our data shows that the calculated spectrum in Figure. $6 \mathrm{~b}$ are very specific to the ErTPP-(acac) in which the role of the apical ligand is non-negligible. Hence, these results confirm our findings in which the ErTPP-(acac) can be switched between the two described adsorbed conformations.

\section{CONCLUSIONS}

We have shown that ErTPP-(acac) molecules can be synthesized and adsorbed on an semiinsulating layer made of $\mathrm{CaF}_{2} / \mathrm{Si}(100)$ at low temperature $(12 \mathrm{~K})$. Our findings indicate that after 
adsorption, some of the adsorbed ErTPP-(acac) molecular conformations involves the Er atom to be pushed towards the surface with the acac ligand strapped against the porphyrin macrocycle. In this configuration, the ligand is slightly unstable under normal scanning conditions. The application of larger excitation voltage on this molecular conformation can stabilize the ErTPP-(acac) and show specific signatures in the $\mathrm{dI} / \mathrm{dV}$ spectrum that can be attributed to a repositioning of the acac ligand in a vertical direction. This process is combined with the movement of the central Er atom and the observation of a specific vibrational spectrum of the molecule in the differential conductance data.

\section{ASSOCIATED CONTENT \\ Supporting information}

The supporting information is available free of charge at :

Details of the mass spectrum analysis of the fabricated molecule. Comparison of the calculated constant height image of the ErTPP conformation A with the measured STM image. Detailed description of the two observed conformations A and B. Details of ten calculated vibrational modes and energies of the ErTPP-(acac) molecule in the conformation B.

\section{AUTHOR INFORMATION}

\section{Corresponding Author}

Damien Riedel - Institut des Sciences Moléculaires d'Orsay(ISMO), CNRS, Univ. Paris Sud, UniversitéParis-Saclay, F-91405 Orsay, France.

orcid.org/0000-0002-7656-5409

*Email: damien.riedel@universite-paris-saclay.fr

\section{Authors}

Yitao Wang Institut des Sciences Moléculaires d'Orsay(ISMO), CNRS, Univ. Paris Sud, UniversitéParis-Saclay, F-91405 Orsay, France.

Orcid No: 0000-0002-7584-3470

Eric Duverger - Institut FEMTO-ST, Univ. Bourgogne Franche-Comté, CNRS, 15B avenue des Montboucons, F -25030 Besançon, France.

Orcid No: 0000-0002-7777-8561

Hélène Sauriat-Dorizon - Institut de Chimie Moléculaire et desMatériaux d'Orsay (ICMMO), CNRS, Univ. Paris Sud,UniversitéParis-Saclay, F-91405 Orsay, France.

Orcid No: 0000-0002-5162-4732 


\section{Author contributions}

All the authors have participated in the writing of the article. D.R and YW performed the experimental measurements and the analysis. DR realized the DFT simulations of the ErTPP-(acac) molecule in the gas phase. H.D. performed the chemical synthesis. ED performed the DFT simulations on the large atomic slab with the ErTPP-(acac) molecule.

\section{CONFLICT OF INTERESTS}

The authors declare no conflicts of interest.

\section{ACKNOWLEDGEMENT}

All the authors wish to thank the French National Agency for Research (ANR) for their financial support of the CHACRA project under the contract number ANR-18-CE30-0004-01. Calculations were performed with the supercomputer regional facility Mesocenter of the University of Franche-Comté as well as the HPC of the LUMAT fédération.

\section{FIGURES CAPTIONS}

Figure 1: Top (a) and (b) side balls-and-stick representations of the ErTPP-(acac) molecule with one apical acetylacetone ligand. The grey, red, white, green and blue balls represent the carbon, oxygen, hydrogen, erbium and nitrogen atoms, respectively. (c) and (d) $\left(22 \times 22 \AA^{2}\right)$ STM topography $\left(V_{\mathrm{s}}=-\right.$ $2.5 \mathrm{~V}, \mathrm{I}=18 \mathrm{pA}$ ) of two ErTPP-(acac) conformations A and B, respectively, observed after adsorption on the $\mathrm{CaF}_{2} / \mathrm{Si}(100)$ surface stripes.

Figure 2: (a) $22 \times 22 \mathrm{~A}^{2}$ STM topography of the ErTPP molecule in the conformation A. The dots indicate where the $\mathrm{dI} / \mathrm{dV}$ spectroscopy curves have been acquired. (b) to (d) $\mathrm{dI} / \mathrm{dV}$ curves acquired on 
the four phenyls (b), the four pyrroles (c) and the central (d) positions on the ErTPP molecule as detailed in (a).

Figure 3: $63 \times 63 \mathrm{~A}^{2}$ constant height mode STM images acquired on top of a ErTPP molecule in the conformation A at a constant bias of -2 Volts. The height of the STM tip apex is 0.5 A higher than the one fixed by the setting parameters $(\mathrm{Vs}=-2.5 \mathrm{~V}, \mathrm{I}=18 \mathrm{pA})$. The dotted lines are placed to help positioning the phenyls rings as well as the center of the molecule.

Figure 4: (a) $22 \times 22 \mathrm{~A}^{2}$ STM topography of the ErTPP molecule in the conformation B. The dots indicate where the $\mathrm{dI} / \mathrm{dV}$ spectroscopy curves have been acquired. (b) to (d) dI/dV curves acquired on the four phenyls (b), the four pyrroles (c) and the central (d) positions on the ErTPP molecule as detailed in (a). Typical maximums of the $\mathrm{dI} / \mathrm{dV}$ peaks are indicated with black arrows.

Figure 5: (a) and (b) projected density of states (PDOS) curves calculated for the two conformations B and A respectively. The corresponding conformations are recalled in the insert of each set of curves. In (a), the $\mathrm{dI} / \mathrm{dV}$ curves are integrated over the sum of the four phenyls, four pyrroles, the Er atom and the acac fragment. In (b), the $\mathrm{dI} / \mathrm{dV}$ curves are integrated over the four phenyls, four pyrroles and the Er atom.

Figure 6: (a) $d^{2} I / d V^{2}$ curve calculated from a section of the acquired $d I / d V$ spectrum at the Ce position on the conformation B of the ErTPP-(acac) molecule from Fig. $4 \mathrm{~d}$. The origin of the curve $(0 \mathrm{~cm}-1)$ is set at the resonance energy maximum of the DOS peak observed at $-1.3 \mathrm{eV}$. (b) Calculated vibrational spectra of the ErTPP-(acac) as in the conformation B in the gas phase (see method). The calculated energy values printed in blue are the most comparable to the ones measured in (a).

\section{REFERENCES}

${ }^{1}$ Katoh, K .; Komeda, T.; Yamashita, M. Surface morphologies, electronic structures, and Kondo effect of lanthanide(III)-phthalocyanine molecules on $\mathrm{Au}(111)$ by using STM, STS and FET properties for next generation devices Dalton transactions. Dalton Trans. 2010, 39, 4708-4723.

${ }^{2}$ Bulach, V.; Sguerra, F.; Hosseini, W. Porphyrin lanthanide complexes for NIR emission. Coordination Chemistry reviews 2012, 256, 1468-1478.

${ }^{3}$ Zhang, Y.; Wang, Y.; Liao, P.: Wang, K.; Huang, Z.; Liu, J.; Chen, Q.; Jiang, J.; Wu, K. Detection and manipulation of Charge States for Double-Decker DyPc2 Molecules on Ultrathin CuO Films. ACS Nano 2018, 12, 2991-2997. 
${ }^{4}$ He, Y.; Zhang, Y. J.; Hong, I. P.; Cheng, F.; Zhou, X.; Shen, Q.; Li, J. L.; Wang, Y. F.; Jiang, J. Z.; Wu, K. LowTemperature Scanning Tunneling Microscopy Study of Double-Decker DyPc2 on Pb Surface. Nanoscale 2014, 6, 10779-10783.

${ }^{5}$ Katoh, K.; Yoshida, Y.; Yamashita, M.; Miyasaka, H.; Breedlove, B. K.; Kajiwara, T.; Takaishi, S.; Ishikawa, N.; Isshiki, H.; Feng, Y. Direct Observation of Lanthanide(III)-Phthalocyanine Molecules on Au(111) by Using Scanning Tunneling Microscopy and Scanning Tunneling Spectroscopy and Thin-Film Field-Effect Transistor Properties of Tb(III)- and Dy(III)-Phthalocyanine Molecules. J. Am. Chem. Soc. 2009, 131, 9967-9976.

${ }^{6}$ Tuerhong, R.; Ngassam, F.; Watanabe, S.; Onoe, J.; Alouani, M.; Bucher, J.-P. Two-Dimensional Organometallic Kondo Lattice with Long-Range Antiferromagnetic Order. J. Phys. Chem. C 2018, 122, 20046-20054.

${ }^{7}$ Fahrendorf, S.; Atodiresei, N.; Besson, C.; Caciuc, V.; Matthes, F.; Blugel, S.; Kogerler, P.; Burgler, D. E.; Schneider, C. M. Accessing 4f-states in single-molecule spintronics. Nat. Commun. 2013, 4, 2425.

${ }^{8}$ Warner, B.; Hallak, F. E.; Atodiresei, N.; Seibt, P.; Pru“ser, H.; Caciuc, V.; Waters, M.; Fisher, A. J.; Blu"gel, S.; Slageren, J. V.; Hirjibehedin, C. F. Sub-molecular modulation of a $4 f$ driven Kondo resonance by surface-induced asymmetry. Nat. Commun. 2016, 7, 12785.

${ }^{9}$ Liao, M.-S.; Scheiner, S. Electronic structure and bonding in metal porphyrins, metal=Fe, Co, Ni, Cu, Zn. J. Chem. Phys. 2002, 117, 205-219.

${ }^{10}$ Orti, E.; Bredas, J. L. Electronic structure of phthalocyanines: Theoretical investigation of the optical properties of phthalocyanine monomers, dimers, and crystals. J. Chem. Phys. 1990, 92, 1228-1235.

${ }^{11}$ de la Torre, G.; Claessens, C. G.; Torres, T. Phthalocyanines: Old Dyes, New Materials. Putting Color in Nanotechnology. Chem. Commun. 2007, 20, 2000-2015.

${ }^{12}$ Reecht, G.; Krane, N.; Lotze, C.; Franke, K. J. $\pi$-Radical Formation by Pyrrolic H Abstraction of Phthalocyanine Molecules on Molybdenum Disulfide. ACS Nano 2019, 13, 7031-7035.

${ }^{13}$ Schwarz, F.; Wang, Y. F.; Hofer, W. A.; Berndt, R.; Runge, E.; Kröger, J. Electronic and Vibrational States of Single TinPhthalocyanine Molecules in Double Layers on Ag(111). J. Phys. Chem. C 2015, 119, 15716-15722.

${ }^{14}$ Mironov, A. F. Lanthanide porphyrin complexes. Russ. Chem. Rev. 2013, 82, 333-351.

${ }^{15}$ Garzon-Roman, A.; Cuate-Gomez, D. H.; Zuiga-Islas, C.; Sosa-Sanchez, J. L.; Lopez-Huerta, F. Erbium phthalocyanine on porous silicon: Morphological, optical, and electrical characterization, for its possible application as a photodetector. Sens. Actuators A 2020, 315, 112309.

${ }^{16}$ He. H. S.; Sykes, A. G. Facile preparation of neutral monoporphyrinate lanthanide complexes with strong nearinfrared emission. Inorg. Chem. Commun. 2008, 11, 1304-1307.

${ }^{17}$ Spyroulias, G. A.; Despotopoulos, A. P.; Raptopoulou, C. P.; Terzis, A.; de Montauzon, D.; Poiblanc, R.; Coutsolelos, A. G. Comparative Study of Structure-Properties Relationship for Novel $\beta$-Halogenated Lanthanide Porphyrins and Their Nickel and Free Bases Precursors, as a Function of Number and Nature of Halogens Atoms. Inorg. Chem. 2002, 41, 2648-2659.

${ }^{18}$ Duverger, E.; Boyer, A. G.; Sauriat-Dorizon, H.; Sonnet, P.; Stephan, R.; Hanf, M.-C.; Riedel, D. TwoDimensional Functionalized Ultrathin Semi-Insulating $\mathrm{CaF}_{2}$ Layer on the $\mathrm{Si}(100)$ Surface at a Low Temperature for Molecular Electronic Decoupling. ACS Appl. Mater. Interfaces 2020, 12, 29661-29670.

${ }^{19}$ Chiaravalloti, F.; Dujardin G.; Riedel, D. Atomic scale control of hexaphenyl molecules manipulation along functionalized ultra-thin insulating layer on the $\operatorname{Si}(100)$ surface at low temperature $(9 \mathrm{~K})$. J. Phys.: Condens. Matter 2015, 27, 054006. 
${ }^{20}$ Nazin, G.V.; Wu, S.W.; Ho, W. Tunneling rates in electron transport through double-barrier molecular junctions in a scanning tunneling microscope. Proc. Natl. Acad. Sci. U. S. A. 2005, 102, 8832-8837.

${ }^{21}$ Hohenberg, P.; Kohn W. Inhomogeneous Electron Gas. Phys. Rev. 1964, 136, 864-871.

${ }^{22}$ Kohn, W.; Sham, L.J. Self-Consistent Equations Including Exchange and Correlation Effects. Phys. Rev. 1965, $140,1133-1138$.

${ }^{23}$ Ordejon, P.; Artacho, E; Soler, J.M. Self-consistent order-N density-functional calculations for very large systems. Phys. Rev. B 1996, 53, 10441-10444.

${ }^{24}$ Soler, J.M.; Artacho, E.; Gale, J.D.; García, A.; Junquera, J.; Ordejón, P.; Sánchez-Portal, D. The SIESTA method for ab initio order- $N$ materials simulation. Journal of Physics: Condensed Matter 2002, 14, 2745-2779.

${ }^{25}$ Pizzoferrato,R.; Francini, R.; Pietrantoni, S.; Paolesse, R.; Mandoj, F.; Monguzzi, A.; Meinardi, F. Effects of progressive halogen substitution on the photoluminescence properties of an erbium-porphyrin complex. J. Phys. Chem. A 2010, 114,4163-8.

${ }^{26}$ Harrison, B. S.; Foley, T. J.; Bouguettaya, M.; Boncella, J. M.; Reynolds, J. R.; Schanze, Kirk S. Near-infrared electroluminescence from conjugated polymer/lanthanide porphyrin blends. Appl. Phys. Lett. 2001, 79, 3770.

${ }^{27}$ Dion, M. ; Rydberg, H.; Schröder, E.; Langreth, D. C.; Lundqvist, B. I. Van der Waals Density Functional for General Geometries. Phys. Rev. Lett. 2004, 92, 246401.

${ }^{28}$ Perdew, J. P.; Burke, K.; Ernzerhof, M. Generalized Gradient Approximation Made Simple. Phys. Rev. Lett. 1996, 77, 3865-3868.

${ }^{29}$ Román-Pérez, G.; Soler, J. M. Efficient Implementation of a van der Waals Density Functional: Application to Double-Wall Carbon Nanotubes. Phys. Rev. Lett. 2009, 103, 096102.

${ }^{30}$ Bader, R. F. W. A quantum theory of molecular structure and its applications Chem. Rev. 1991, 91, 893-928.

${ }^{31}$ Henkelman, G.; Arnaldsson, A.; Jońsson, H. A fast and robust algorithm for Bader decomposition of charge density. Comput. Mater. Sci. 2006, 36, 354-360.

${ }^{32}$ Labidi, H.; Pinto, P.; Leszczynski, L.; Riedel, D. Exploiting a single intramolecular conformational switch to probe charge transfer dynamics at the nanoscale. Phys. Chem. Chem. Phys. 2017, 19, 28982.

${ }^{33}$ Ramos, P.; Mankarious, M.; Pavanello, M.; Riedel, D. Probing Charge Transfer Dynamics in a Single Iron Tetraphenylporphyrin Dyad Adsorbed on an Insulating Surface. Nanoscale 2018, 10, 17603-17616.

${ }^{34}$ Huan, Q.; Jiang, Y.; Zhang, Y. Y.; Ham, U.; Ho, W. Spatial Imaging of Individual Vibronic States in the Interior of Single Molecules. J. Chem. Phys. 2011, 135, 014705.

${ }^{35}$ Krane, N.; Lotze, C.; Reecht, G.; Zhang, L.; Briseno, A. L.; Franke, K. J. High-Resolution Vibronic Spectra of Molecules on Molybdenum Disulfide Allow for Rotamer Identification. ACS Nano 2018, 12, 11698-11703.

${ }^{36}$ Roy, D. R.; Shah, E. V.; Roy, S. M. Optical activity of Co-porphyrin in the light of IR and Raman spectroscopy: A critical DFT investigation. Spectrochim. Acta, Part A 2018, 190, 121-128. 


\section{TOC GRAPHIC}

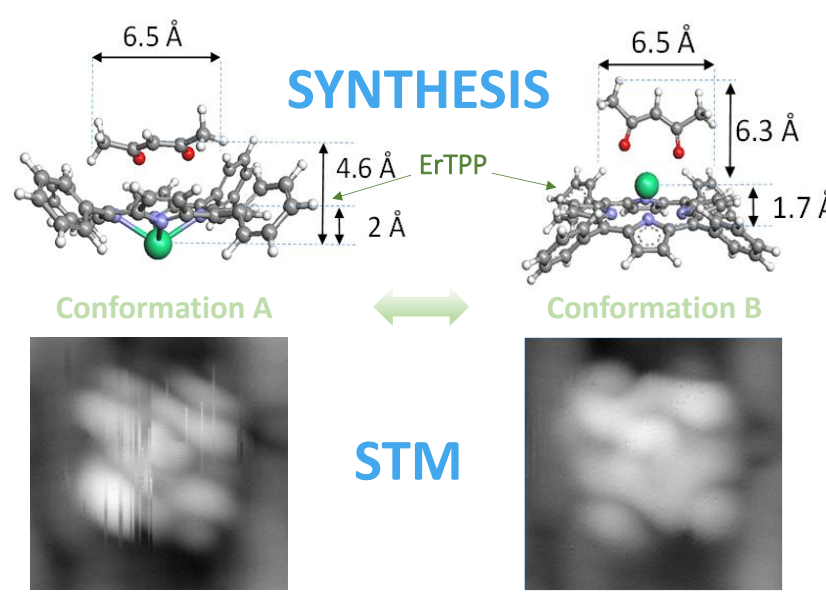

